\title{
LUCAS PARTITIONS
}

\author{
NEVILLE ROBBINS \\ Mathematics Department \\ San Francisco State University \\ San Francisco, CA 94132, U.S.A.
}

(Received April 1, 1996)

\begin{abstract}
The Lucas sequence is defined by: $L_{0}=2, L_{1}=1, L_{n}=L_{n-1}+L_{n-2}$ for $n \geq 2$. Let $V(n), r(n)$ denote respectively the number of partitions of $n$ into parts, distinct parts from $\left\{L_{n}\right\}$. We develop formulas that facilitate the computation of $V(n)$ and $r(n)$.
\end{abstract}

\section{KEY WORDS AND PHRASES:}

1991 AMS SUBJECT CLASSIFICATION CODES: 11P81, 11P83, $11 \mathrm{~B} 35$.

\section{INTRODUCTION}

Let $S$ denote a non-empty subset of $N$, the set of all natural numbers. Let $V(n), r(n), r_{E}(n)$, $r_{0}(n)$ denote respectively the number of partitions of $n$ into parts, distinct parts, evenly many distinct parts, oddly many distinct parts from $S$. Define $V(0)=r(0)=r_{E}(0)=1, r_{0}(0)=0$. Let $V(n)$ have the generating function:

$$
F(z)=\sum_{n=0}^{\infty} V(n) z^{n}
$$

Let

$$
1 / F(z)=\sum_{n=0}^{\infty} a(n) z^{n}
$$

It follows from (1.1) and (1.2) that

$$
\sum_{k=0}^{n} a(n-k) V(k)=0 \text { for } n \geq 1 .
$$

Furthermore,

$$
a(n)=r_{E}(n)-r_{0}(n)
$$

REMARK. Apostol [1], p.311 and Hardy [3], p.285 prove that (1.4) holds when $S=N$, but the same reasoning applies to the more general case. Since also

$$
r(n)=r_{E}(n)+r_{0}(n)
$$

it follows that

$$
a(n)=2 r_{E}(n)-r(n)=r(n)-2 r_{0}(n) .
$$

In this note, we consider the case where $S$ is the set of all Lucas numbers, $L_{n}$, where $n \geq 0$. (The Lucas numbers are defined by: $L_{0}=2, L_{1}=1, L_{n}=L_{n-1}+L_{n-2}$ if $n \geq 2$.) We will show how to compute the $r(n)$ and the $a(n)$ : via explicit formulas if $n=L_{k}$ or $n=1+L_{2 k+1}$ for some $k$, recursively otherwise. The $V(n)$ can then be computed recursively via (1.3). 


\section{PRELIMINARIES}

Notation and Definitions

$F_{k}=k^{\text {th }}$ Fibonacci number $\left(F_{0}=0, F_{1}=1, F_{k}=F_{k-1}+F_{k-2}\right.$ if $\left.k \geq 2\right)$

$L_{k}=k^{\text {th }}$ Lucas number $\left(L_{0}=2, L_{1}=1, L_{k}=L_{k-1}+L_{k-2}\right.$ if $\left.k \geq 2\right)$

$E(n)=\sum_{k=1}^{n}|a(k)|$

$[x]$ denotes the integer part of the real number $x$

$[a, b]$ denotes the set of all integers, $t$, such that $a \leq t \leq b$.

In particular, if $k \geq 3$, then

$$
\begin{aligned}
I_{k} & =\left[L_{k+1}, L_{k+2}-1\right] \\
I_{k, 1} & =\left[L_{k+1}, 2 L_{k}-1\right] \\
I_{k, 2} & =\left[2 L_{k}, 5 F_{k}-1\right] \\
I_{k, 3} & =\left[5 F_{k}, L_{k+2}-1\right]
\end{aligned}
$$

\section{Lucas Identities}

(1) $L_{n}=L_{n-1}+L_{n-2}$ for $n \geq 2$, with $L_{0}=2, L_{1}=1$

(2) $L_{j}<L_{k}$ iff $j<k$, unless $j=k-1=0$

(3) $\left\{L_{n}\right\}$ is strictly increasing if $n \geq 1$

(4) $L_{n}=\alpha^{n}+\beta^{n}$, where $\alpha=\frac{1}{2}\left(1+5^{\frac{1}{2}}\right), \beta=\frac{1}{2}\left(1-5^{\frac{1}{2}}\right)$

(5) $L_{2 n}=L_{n}^{2}-2(-1)^{n}$

(6) $L_{n}>1.6^{n}$ if $n \geq 4$

(7) $\quad \sum_{i=0}^{k+1} L_{\imath}=L_{k+3}-1$

(8) $\sum_{i=0}^{\left[\frac{1}{2} \jmath\right]} L_{\jmath-2 \mathrm{z}}=L_{\jmath+1}+t$, where $t=\left\{\begin{aligned} 1 & \text { if } 2 / j \\ -2 & \text { if } 2 \chi_{j}\end{aligned}\right.$

(9) $\mathrm{L}_{n+1}+L_{n-1}=5 F_{n}$

(10) $L_{k+2}-5 F_{k}=2 L_{k}-L_{k+1}=L_{k-2}$

REMARKS. (1) is the definition of the Lucas sequence (2) and (3) follow from (1). (4) follows from (1), using induction and the fact that $\alpha, \beta$ are the roots of $u^{2}-u-1=0$. (5) and (6) follow from (4). (7) through (9) may be proved using induction on $n$. (10) follows from (1) and (9).

\section{THE MAIN THEOREMS}

Let $n$ be a natural number. We first address the issue of the representability of $n$ as a sum of distinct Lucas numbers. Such a representation will be called a Lucas representation of $n$. If in addition, the summands are non-consecutive Lucas numbers, we say that the Lucas representation of $n$ is special. We will show that every natural number has a special Lucas representation. If the special Lucas representation of $n$ is unique, which is usually the case, we call it the minimal Lucas representation of $n$. Otherwise, $n$ has two special Lucas representations. In this case, we define the minimal Lucas representation of $n$ as the special Lucas representation that does not include $L_{0}=2$ as a summand.

For example, 13 has the unique special (and hence minimal) Lucas representation $13=11+2=L_{5}+L_{0} ; 12$ has two special Lucas representations: $12=11+1=L_{5}+L_{1}$, and $12=7+3+2=L_{4}+L_{2}+L_{0}$. The former is the minimal Lucas representation of 12 .

THEOREM 1. Every natural number, $n$, has a special Lucas representation:

$$
n=L_{k_{1}}+L_{k_{2}}+\text { etc. }+L_{k_{r}}
$$

where $k_{i}-k_{2+1} \geq 2$ for all $i$ such that $1 \leq i \leq r-1$, if $r \geq 2$.

PROOF. (Induction on $n$ ) It suffices to consider the case where $n \neq L_{k}$. Therefore there exists unique $k_{1} \geq 3$ such that $L_{k_{1}}<n<L_{k_{1}+1}$. Let $n_{1}=n-L_{k_{1}}$. Now (1) implies $0<n_{1}<L_{k_{1}-1}$. By induction hypothesis, we have $n_{1}=L_{k_{2}}+L_{k_{3}}+$ etc. $+L_{k_{r}}$, with $r \geq 2$ and $k_{\imath}-k_{\imath+1} \geq 2$ for all $i$ such 
that $2 \leq i \leq r$, if $r \geq 3$. Thus $L_{k_{2}} \leq n_{1}$, hence $L_{k_{2}}<L_{k_{1}-1}$. Since $k_{1} \geq 3$, (2) implies $k_{2}<k_{1}-1$, that is, $k_{1}-k_{2} \geq 2$. Since $n=L_{k_{1}}+n_{1}$, the conclusion now follows.

LEMMA 1. Let $n=L_{\jmath_{1}}+L_{\jmath_{2}}+$ etc. $+L_{\jmath_{s}}$, with $s \geq 2$ and $j_{i}-j_{\imath+1} \geq 2$ for all $i$ such that $1 \leq i \leq s-1$. Let $j=j_{1}$. Then $n \leq L_{j+1}+(-1)^{j}$. Furthermore, $n=L_{j+1}+(-1)^{j}$ iff $s=1+\left[\frac{1}{2} j\right], j_{s}=0$, and $j_{\imath}=j-2(i-1)$ for all $i$ such that $1 \leq i \leq s-1$.

PROOF. Using (2) and our hypothesis, we have $L_{j_{i}} \leq r+L_{\jmath-2(2-1)}$ where $r=\left\{\begin{array}{l}1 \text { if } j_{i}=0 \text { and } j=2 i-1 \\ 0 \text { otherwise }\end{array}\right.$

$$
n=\sum_{\imath=1}^{s} L_{j_{\imath}} \leq r+\sum_{\imath=1}^{s} L_{j-2(\mathfrak{\imath}-1)}=r+\sum_{i=0}^{s-1} L_{j-2 \imath} \leq r+\sum_{i=0}^{\left.\left[\frac{1}{2}\right]\right]} L_{\jmath-2 \imath} .
$$

Now (8) implies $n \leq L_{j+1}+(-1)^{j}$. If $s=1+\left[\frac{1}{2} j\right], j_{s}=0$, and $j_{2}=j-2(i-1)$ for all $i$ such that $1 \leq i \leq s-1$, then the weak inequalities in (3.2) may be replaced with equalities, which yields $n=L_{\jmath+1}+(-1)^{\jmath}$. Conversely, if $n=L_{j+1}+(-1)^{j}$, then the weak inequalities in (3.2) become equalities. This implies $s=1+\left[\frac{1}{2} j\right], L_{j_{1}}=L_{j-2(\imath-1)}$ (and hence $j_{i}=j-2(i-1)$ ) for all $i$ such that $1 \leq i \leq s-1$, and $j_{s}=0$.

LEMMA 2. $L_{\imath}=L_{j}+1$ iff $(i, j)=(0,1),(2,0)$, or $(3,2)$.

PROOF. Suppose $L_{\imath}=L_{j}+1$. If $j=0$, then $L_{\imath}=3$, so $i=2$. If $j=1$ and $i<j$, then $i=0$. Now suppose $i>j \geq 1$. Then (3) and (1) imply $1=L_{i}-L_{\jmath} \geq L_{i}-L_{\imath-1}=L_{\imath-2}$. Therefore $L_{\imath-2}=1$, so $i=3$ and $j=2$. The converse follows by direct substitution.

LEMMA 3. If

$$
n=L_{k}
$$

then this special Lucas representation of $n$ is unique.

PROOF. Let $k$ be the least index such that the special representation (3.3) is not unique. By inspection, $k \geq 4$. Thus $n$ has a second special Lucas representation:

$$
n=L_{\jmath_{1}}+L_{\jmath_{2}}+\text { etc. }+L_{\jmath_{s}}
$$

with $j_{i}-j_{i+1} \geq 2$ for all $i$ such that $1 \leq i \leq s-1$. In fact, (2) implies $s \geq 2$. Let $j=j_{1}$. Now (3.4) implies $L_{j}<n$, so $L_{\jmath}<L_{k}$. If $2 \gamma_{j}$, then Lemma 1 implies $L_{k} \leq L_{\jmath+1}-1$, so $L_{k}<L_{\jmath+1}$ But then $L_{\jmath}<L_{k}<L_{\jmath+1}$, an impossibility. If $2 \mid j$, then Lemma 1 implies $L_{k} \leq L_{\jmath+1}+1$. Since $k \geq 4$, Lemma 2 implies $L_{k} \neq L_{\jmath+1}+1$. Therefore $L_{k}<L_{\jmath+1}+1$, so that $L_{k} \leq L_{j+1}$. Since $L_{\jmath}<L_{k} \leq L_{j+1}$, we must have $L_{k}=L_{\jmath+1}$, hence $k=j+1$. Now (3.4) yields $L_{k}=L_{k-1}+L_{\jmath_{2}}+$ etc. $+L_{\jmath_{s}}$, hence $L_{k-2}=L_{j_{2}}+$ etc. $+L_{j_{s}}$. By definition of $k$, we must have $s=2, j_{2}=k-2$. But then $j_{1}-j_{2}=1$, an impossibility.

THEOREM 2. Let $n$ have two distinct special Lucas representations:

$$
\begin{array}{ll}
n=L_{k_{1}}+L_{k_{2}}+\text { etc. }+L_{k_{r}} & \begin{array}{l}
\text { with } k_{i}-k_{i+1} \geq 2 \text { for all } i \\
\text { such that } 1 \leq i \leq r-1
\end{array} \\
n=L_{\jmath_{1}}+L_{\jmath_{2}}+\text { etc. }+L_{\jmath_{s}} & \begin{array}{l}
\text { with } j_{i}-j_{2+1} \geq 2 \text { for all } i \\
\text { such that } 1 \leq i \leq s-1 .
\end{array}
\end{array}
$$

Assume also that $j=j_{1}<k_{1}$. Then $k_{1}=2 s-1, k_{2}=k_{r}=1$, and $j_{i}=2(s-i)$ for all $i$ with $1 \leq i \leq s$.

PROOF. Let $k_{1}=k$. Note that Lemma 3 implies $\operatorname{Min}\{r, s\} \geq 2$. Thus $n \geq 5$ and $j \geq 2$. Let $j=k-m$, where $m \geq 1$. Lemma 1 implies $n \leq L_{\jmath+1}+(-1)^{\jmath}=L_{k-m+1}+(-1)^{k-m}$. By hypothesis, $L_{k}<n$, so that $L_{k}<L_{k-m+1}+(-1)^{k-m}$. If $m \geq 2$, then $L_{k-m+1} \leq L_{k-1}$ by (2), since if $k=2$, then $j=1=s$. Thus $L_{k}<L_{k-1}+(-1)^{k-m}$, which implies $L_{k-2}<1$, an impossibility, since $L_{n} \geq 1$ for all $n$. Therefore $m=1$, so $0<(-1)^{k-1}$ implies $k$ is odd. Since $L_{k}<n \leq L_{k}+1$, we 
must have $n=L_{k}+1=L_{k}+L_{1}$, so $k_{2}=k_{r}=1$. Now (3.6) and Lemma 1 imply $j_{2}=2(s-i)$ for all $i$ such that $1 \leq i \leq s$.

THEOREM 3. Let $n$ have the special Lucas representation:

$$
n=L_{k_{1}}+L_{k_{2}}+\text { etc. }+L_{k_{r}} \text {. }
$$

This special Lucas representation is unique unless $k_{r}=1$ and $k_{r-1}=2 h+1$ for some $h \geq 1$, in which case $n$ has a second special Lucas representation:

$$
n=L_{k_{1}}+L_{k_{2}}+\text { etc. }+L_{k_{r-2}}+L_{2 h}+L_{2 h-2}+\text { etc. }+L_{2}+L_{0} .
$$

PROOF. If $r=1$, then the special Lucas representation (3.7) is unique by Lemma 3 If $r \geq 2$, suppose that $n$ has a second special Lucas representation:

$$
n=L_{j_{1}}+L_{\jmath_{2}}+\text { etc. }+L_{\jmath_{s}} .
$$

Again, by Lemma 3,s $\geq 2$. If $j_{1}<k_{1}$, then the conclusion follows from Theorem 2, with $r=2$ and $h=s$. Now suppose that $j_{\imath}=k_{i}$ for all $i$ such that $1 \leq i \leq u-1$ (for some $u \geq 2$ ), but $j_{u}<k_{u}$. Let

$$
m=n-\sum_{i=1}^{u-1} L_{k_{1}}=L_{k_{u}}+L_{k_{u+1}}+\text { etc. }+L_{k_{r}}
$$

also

$$
m=n-\sum_{i=1}^{u-1} L_{\jmath_{i}}=L_{\jmath_{u}}+L_{j_{u+1}}+\text { etc. }+L_{\jmath_{s}} .
$$

Now Theorem 2 implies $j_{u+i}=2(s-u-i)$ for all $i$ such that $0 \leq i \leq s-u, u=r-1$, $k_{u}=k_{r-1}=2(s-u)-1=2(s-r)+1, k_{r}=1$. The conclusion now follows from Theorem 2 , with $h=s-r$.

Combining the results of Theorems 1,2 , and 3 , we have:

THEOREM 4. Every natural number, $n$, has a unique minimal Lucas representation:

$$
n=L_{k_{1}}+L_{k_{2}}+\ldots+L_{k_{r-1}}+L_{k_{r}}
$$

where (i) $k_{\imath}-k_{i+1} \geq 2$ for all $i$ such that $1 \leq i \leq r-1$, if $r \geq 2$; (ii) if $r \geq 2$ and $k_{r}=0$, then $k_{r-1} \geq 3$.

LEMMA 4. Let $n$ have the minimal Lucas representation given by (3.10) in Theorem 4 above. Then $L_{k_{1}}<n<L_{k_{1}+1}$, if $r \geq 2$.

PROOF. (Induction on $r$ ) Clearly, $L_{k_{1}}<n$, so it suffices to show that $\hbar<L_{k_{1}+1}$. Let $r=2$, so $n=L_{k_{1}}+L_{k_{2}}$. If $k_{2} \geq 1$, then by hypothesis, $k_{2} \leq k_{1}-2$, so (2) implies $L_{k_{2}} \leq L_{k_{1}-2}<L_{k_{1}-1}$. Thus $n \leq L_{k_{1}}+L_{k_{1}-2}<L_{k_{1}}+L_{k_{1}-1}=L_{k_{1}+1}$. If $k_{2}=0$, then by (1) and (2), we have $n=L_{k_{1}}+L_{k_{2}}=L_{k_{1}}+L_{0}=L_{k_{1}}+2<L_{k_{1}}+3=L_{k_{1}}+L_{2} \leq L_{k_{1}}+L_{k_{1}-1}=L_{k_{1}+1}$, so $n<L_{k_{1}+1}$. If $r \geq 3$, let $n_{1}=n-L_{k_{1}}=L_{k_{2}}+$ etc. $+L_{k_{r}}$. Clearly, this is a minimal Lucas representation of $n_{1}$, so by induction hypothesis, we have $n_{1}<L_{k_{2}+1}$, hence $n<L_{k_{1}}+L_{k_{2}+1}$. Since $1 \leq k_{2}+1 \leq k_{1}-1$ by hypothesis, (2) implies $L_{k_{2}+1} \leq L_{k_{1}-1}$. Therefore $n<L_{k_{1}}+L_{k_{1}-1}=L_{k_{1}+1}$.

The three following theorems permit the computation of $r\left(L_{n}\right)$ and $a\left(L_{n}\right)$.

THEOREM 5. $r\left(L_{n}\right)=\left[\frac{1}{2}(n+2)\right]$ if $n \geq 0$.

PROOF. (Induction on $n$ ) The statement is true by inspection if $n=0$ or 1 . If $n \geq 2$, and if $L_{n}$ is partitioned into several distinct parts, then (7) implies that the largest part must be $L_{n-1}$. Therefore, by (1), we have $r\left(L_{n}\right)=1+r\left(L_{n-2}\right)=1+\left[\frac{1}{2} n\right]$ (by induction hypothesis) $=\left[\frac{1}{2}(n+2)\right]$. (The "1" in the last equation arises from the trivial partition: $L_{n}=L_{n}$.)

THEOREM 6. $r_{E}\left(L_{n}\right)=\left[\frac{1}{4}(n+2)\right]$ if $n \geq 0$. 
PROOF. (Induction on $n$ ) The statement is true by inspection if $n=0$ or 1 . If $n \geq 2$, then reasoning as in the proof of Theorem 5, we have $r_{E}\left(L_{n}\right)=r_{0}\left(L_{n-2}\right)=r\left(L_{n-2}\right)-r_{E}\left(L_{n}-2\right)=$ $\left[\frac{1}{2} n\right]-\left[\frac{1}{4} n\right]=\left[\frac{1}{4}(n+2)\right]$ by Theorem 5 and induction hypothesis.

THEOREM 7. $a\left(L_{n}\right)=\left\{\begin{aligned} 0 & \text { if } n \equiv 2,3(\bmod 4) \\ -1 & \text { if } n \equiv 0,1(\bmod 4)\end{aligned}\right.$.

PROOF. This follows from (1.6) and from Theorems 5 and 6.

Having settled the case where $n$ is a Lucas number, we now consider the case where $n$ is a sum of two or more distinct, non-consecutive Lucas numbers. Then, by Theorem $4, n$ has a unique minimal Lucas representation:

$$
n=\sum_{k=1}^{r} L_{k_{1}}
$$

where $r \geq 2, k_{2}-k_{i+1} \geq 2$ for all $i$ such that $1 \leq i \leq r-1$, and if $k_{r}=0$, then $k_{r-1} \geq 3$.

Alternatively, we could write:

$$
n=\sum_{j=0}^{s} c_{j} L_{j}
$$

where (i) $c_{s}=1$; (ii) $c_{\jmath}=0$ or 1 for all $j$ such that $0 \leq j \leq s-1$; (iii) $c_{\jmath-1} c_{j}=0$ for all $j$ such that $1 \leq j \leq s$; (iv) if $c_{0}=1$, then $c_{2}=0$.

If we omit the conditions (iii) and (iv), then (3.12) corresponds to a Lucas representation of $n$. The $c$, will be called the digits of the representation.

Referring again to (3.11), let $n_{1}=n-L_{k_{1}}>0, n_{2}=n_{1}-L_{k_{2}} \geq 0$. Given any Lucas representation of $n$, define the initial segment as the first $k_{1}-k_{2}$ digits; define the terminal segment as the remaining digits. In the minimal Lucas representation of $n$, the initial segment consists of a 1 followed by $k_{1}-k_{2}-10$ 's, and corresponds to the minimal Lucas representation of $L_{k_{1}-k_{2}-1}$, while the terminal segment corresponds to the minimal Lucas representation of $n_{1}$. Lucas representations of $n$ may be obtained as follows:

Type I. Arbitrary combinations of Lucas representations of the integers corresponding to the initial and terminal segments in the minimal Lucas representation of $n$, namely $L_{k_{1}-k_{2}-1}$ and $n_{1}$. Clearly, the number of Type I Lucas representations of $n$ is $r\left(L_{k_{1}-k_{2}-1}\right) r\left(n_{1}\right)=\left[\frac{1}{2}\left(k_{1}-k_{2}+1\right)\right] r\left(n_{1}\right)$.

Type II. Suppose that in a non-minimal Lucas representation of $n$, the initial segment ends in 10, while the terminal segment starts with 0 . If this block of digits, consisting of 100 , is replaced by 011 , then a new Lucas representation of $n$ is obtained. A necessary condition for the existence of Type II Lucas representations is that $2 \mid\left(k_{1}-k_{2}\right)$.

Type III. In the minimal Lucas representation of $n$, if $k_{r}=1$ and $k_{r-1}=2 h+1$ for some $h \geq 1$, then by Theorem 3, a new Lucas representation of $n$ is obtained by replacing $L_{2 h+1}+L_{1}$ by $L_{2 h}+L_{2 h-2}+$ etc. $+L_{2}+L_{0}$.

The three following theorems enable us to compute $r\left(1+L_{2 k+1}\right)$ and $a\left(1+L_{2 k+1}\right)$.

THEOREM 8. If $k \geq 1$, then $r\left(L_{2 k+1}+1\right)=k+1$.

PROOF. Let $n=L_{2 k+1}+1=L_{2 k+1}+L_{1}$. Here $n_{1}=L_{1}$, so the number of Type I Lucas representations of $n$ is $r\left(L_{2 k-1}\right) r\left(L_{1}\right)=\left[\frac{2 k+1}{2}\right]\left[\frac{3}{2}\right]=k$. Since $L_{1}$ has no Lucas representation but the minimal one, there are no Type II Lucas representations of $n$. By hypothesis and Theorem 3 , there is a unique Type III Lucas representation of $n$. Therefore $r\left(L_{2 k+1}+1\right)=k+1$.

THEOREM 9. If $k \geq 1$, then $r_{E}\left(L_{4 k+1}+1\right)=k ; r_{E}\left(L_{4 k-1}+1\right)=k+1$.

PROOF. Let $n=L_{2 j+1}+1=L_{2 j+1}+L_{1}$. As in the proof of Theorem 8, $n$ has no Type II Lucas representations. A Type I Lucas representation has an even number of terms iff its initial segment has an odd number of terms. Therefore the number of such Type I Lucas representations of $n$ is $r_{0}\left(L_{2 j-1}\right)=r\left(L_{2 j-1}\right)-r_{E}\left(L_{2 j-1}\right)=\left[\frac{1}{2}(2 j+1)\right]-\left[\frac{1}{4}(2 j+1)\right]=j-\left[\frac{1}{4}(2 j+1)\right]$ by $(1.5)$ and Theorems 5 and 6 . Whether $j=2 k$ or $2 k-1$, the number of Type I Lucas representations of $n$ with evenly many terms is $k$, since $2 k-\left[\frac{1}{4}(4 k+1)\right]=k=(2 k-1)-\left[\frac{1}{4}(4 k-1)\right]$. The unique Type III 
Lucas representation of $n$ has $j+1$ terms, and thus contributes to $r_{E}\left(L_{2 j+1}+1\right)$ iff $j$ is odd. The conclusion now follows.

THEOREM 10. If $k \geq 1$, then $a\left(L_{4 k+1}+1\right)=-1 ; a\left(L_{4 k-1}+1\right)=2$.

PROOF. This follows from (1.6) and from Theorems 8 and 9.

In Theorems 11, 12, and 13 below, we develop formulas for $r(n), r_{E}(n)$, and $a(n)$ in the case where $n \neq L_{k}, n \neq L_{2 k+1}+1$. In order to do so, we must be able to count the number of Type II Lucas representations of $n$. We therefore need to determine the number of Lucas representations of $n$ that do not include the largest possible Lucas number as a part. This question is addressed by Lemma 5 .

LEMMA 5. Let $n$ have the minimal Lucas representation:

$$
n=L_{k_{1}}+L_{k_{2}}+\text { etc. }+L_{k_{r}} \text {. }
$$

Let $n_{1}=n-L_{k_{1}} \geq 0$. Let $\bar{r}(n)$ denote the number of Lucas representations of $n$ that do not include $L_{k_{1}}$ as a part; let $r_{E}(n), r_{0}(n)$ denote respectively the number of such representations consisting of evenly, oddly many parts. Then

$$
\begin{gathered}
\bar{r}(n)=r(n)-r\left(n_{1}\right) \\
\bar{r}_{E}(n)=r_{E}(n)-r_{0}\left(n_{1}\right) \\
\bar{r}_{0}(n)=r_{0}(n)-r_{0}(n)-r_{E}\left(n_{1}\right) .
\end{gathered}
$$

PROOF. It follows from the definitions of $\bar{r}(n), \bar{r}_{E}(n), \bar{r}_{0}(n)$ that $r(n)-\bar{r}(n)$ is the number of Lucas representations of $n$ that do include $L_{k_{1}}$ as a part; $r_{E}(n)-\bar{r}_{E}(n), r_{0}(n)-\bar{r}_{0}(n)$ are respectively the number of such representations consisting of evenly, oddly many parts. If $n \neq L_{k}$ let

$$
n=L_{k_{1}}+L_{j_{2}}+\text { etc. }+L_{j_{s}} \quad(\text { with } s \geq 2)
$$

be a Lucas representation of $n$ that includes $L_{k_{1}}$ as a part. (It follows from Lemma 4 that $L_{k_{1}}$ is the largest part.) Corresponding to (3.16), there is a Lucas representation of $n_{1}$ :

$$
n_{1}=L_{j_{2}}+\text { etc. }+L_{\jmath_{s}} \text {. }
$$

This correspondence is clearly a bijection, so that $r(n)-\bar{r}(n)=r\left(n_{1}\right)$. Furthermore, the number of parts in (3.16) and (3.17) differ in parity. Therefore $r_{E}(n)-\bar{r}_{E}(n)=r_{0}\left(n_{1}\right)$ and $r_{0}(n)-\bar{r}_{0}(n)=r_{E}\left(n_{1}\right)$. The conclusions (3.13), (3.14), (3.15) now follow if $n \neq L_{k}$. If $n=L_{k}$, so that $n_{1}=0$, then clearly no other Lucas representation of $n$ includes $L_{k}$ as a part. Therefore $\bar{r}(n)=r(n)-1=r(n)-r(0)=r(n)-r\left(n_{1}\right)$. Furthermore, $\bar{r}_{E}(n)=r_{E}(n)=r_{E}(n)-0=r_{E}(n)-r_{0}\left(n_{1}\right)$; $\bar{r}_{0}(n)=r_{0}(n)-1=r_{0}(n)-r_{E}\left(n_{1}\right)$.

THEOREM 11. Let $n$ have the minimal Lucas representation:

$$
n=L_{k_{1}}+L_{k_{2}}+\text { etc. }+L_{k_{r}}
$$

where (i) $r \geq 2$; (ii) if $k_{r}=1$, then $2 \mid k_{r-1}$. Let $n_{1}=n-L_{k_{1}}, n_{2}=n_{1}-L_{k_{2}} \geq 0$. Then

$$
r(n)=\left\{\begin{array}{ll}
\frac{1}{2}\left(k_{1}-k_{2}+1\right) r\left(n_{1}\right) & \text { if } 2 \gamma\left(k_{1}-k_{2}\right) \\
\left(1+\frac{1}{2}\left(k_{1}-k_{2}\right)\right) r\left(n_{1}\right)-r\left(n_{2}\right) & \text { if } 2 \mid\left(k_{1}-k_{2}\right)
\end{array} .\right.
$$

PROOF. By hypothesis, there are no Type III Lucas representations of $n$. As mentioned earlier, the number of Type I Lucas representations of $n$ is $\left[\frac{1}{2}\left(k_{1}-k_{2}+1\right)\right] r\left(n_{1}\right)$. If $2 \gamma\left(k_{1}-k_{2}\right)$, then there are no Type II Lucas representations of $n$, so that $r(n)=\left[\frac{1}{2}\left(k_{1}-k_{2}+1\right)\right] r\left(n_{1}\right)=\frac{1}{2}\left(k_{1}-k_{2}+1\right) r\left(n_{1}\right)$. If $2 \mid\left(k_{1}-k_{2}\right)$, then the number of Type II Lucas representations of $n$ is the number of Lucas representations of $n_{1}$ that do not include $L_{k_{2}}$ as a part, namely $\bar{r}\left(n_{1}\right)$. By Lemma 5 , we have $\bar{r}\left(n_{1}\right)=r\left(n_{1}\right)-r\left(n_{2}\right)$. Therefore

$$
r(n)=\left[\frac{1}{2}\left(k_{1}-k_{2}+1\right)\right] r\left(n_{1}\right)+r\left(n_{1}\right)-r\left(n_{2}\right)=\left(1+\frac{1}{2}\left(k_{1}-k_{2}\right)\right) r\left(n_{1}\right)-r\left(n_{2}\right) .
$$


COROLLARY 1. If $n \geq 2$, then $r\left(L_{n}-3\right)=\left[\frac{1}{2} n\right]$.

PROOF. (Induction on $n$ ) By inspection, the conclusion is true if $2 \leq n \leq 5$. If $n \geq 6$, then $L_{n}-3=L_{n-1}+\left(L_{n-2}-3\right)=L_{n-1}+L_{n-3}+\left(L_{n-4}-3\right)$. Now Theorem 11 implies $r\left(L_{n}-3\right)=$ $2 r\left(L_{n-2}-3\right)-r\left(L_{n-4}-3\right)$. By induction hypothesis, we have $r\left(L_{n-2}-3\right)=\left[\frac{1}{2}(n-2)\right]=\left[\frac{1}{2} n\right]-1$, and $r\left(L_{n-4}-3\right)=\left[\frac{1}{2}(n-4)\right]=\left[\frac{1}{2} n\right]-2$. Therefore $r\left(L_{n}-3\right)=2\left(\left[\frac{1}{2} n\right]-1\right)-\left(\left[\frac{1}{2} n\right]-2\right)=\left[\frac{1}{2} n\right]$.

COROLLARY 2. If $n \geq 1$, then $r\left(L_{n}^{2}-1\right)=n$.

PROOF. If $m \geq 1$, then via (5), we have $r\left(L_{2 m}^{2}-1\right)=r\left(L_{4 m}+1\right)=r\left(L_{4 m}+L_{1}\right)$. Now Theorem 11 implies $r\left(L_{2 m}^{2}-1\right)=(2 m) r\left(L_{1}\right)=(2 m) 1=2 m$. again, via (5), we have $r\left(L_{2 m-1}^{2}-1\right)=r\left(L_{4 m-2}-3\right)$. Now Corollary 1 implies $r\left(L_{2 m-1}^{2}\right)=\left[\frac{1}{2}(4 m-2)\right]=2 m-1$.

REMARK. Corollaries 1 and 2 imply (independently) that the function $r(n)$ is a surjection from $N$ to $N$

THEOREM 12. Let $n \neq L_{k}, n \neq L_{2 k+1}+1$. Then

$$
r_{E}(n)=\left\{\begin{array}{ll}
\frac{1}{4}\left(k_{1}-k_{2}+1\right) r\left(n_{1}\right) & \text { if } k_{1}-k_{2} \equiv 3(\bmod 4) \\
\frac{1}{4}\left(k_{1}-k_{2}+3\right) r\left(n_{1}\right)-r_{E}\left(n_{1}\right) & \text { if } k_{1}-k_{2} \equiv 1(\bmod 4) \\
\frac{1}{4}\left(k_{1}-k_{2}+2\right) r\left(n_{1}\right)-r_{0}\left(n_{2}\right) & \text { if } k_{1}-k_{2} \equiv 2(\bmod 4) \\
\left(1+\frac{1}{4}\left(k_{1}-k_{2}\right)\right) r\left(n_{1}\right)-r_{E}\left(n_{1}\right)-r_{E}\left(n_{2}\right) & \text { if } k_{1}-k_{2} \equiv 0(\bmod 4)
\end{array} .\right.
$$

PROOF. By hypothesis and Theorem 3, any Type III Lucas representation of $n$ must arise from a corresponding Type III Lucas representation of $n_{1}=n-L_{k_{1}}$. Thus it suffices to count the Type I and II Lucas representations of $n$ consisting of evenly many parts. A Type I Lucas representation of $n$ with evenly many parts will occur whenever the initial and terminal segments agree in parity. Therefore the number of such representations is given by:

$$
\begin{aligned}
& r_{E}\left(L_{k_{1}-k_{2}-1}\right) r_{E}\left(n_{1}\right)+r_{0}\left(L_{k_{1}-k_{2}-1}\right) r_{0}\left(n_{1}\right)= \\
& \quad\left[\frac{1}{2}\left(k_{1}-k_{2}+1\right)\right] r_{E}\left(n_{1}\right)+\left(\left[\frac{1}{2}\left(k_{1}-k_{2}+1\right)\right]-\left[\frac{1}{4}\left(k_{1}-k_{2}+1\right)\right]\right)\left(r\left(n_{1}\right)-r_{E}\left(n_{1}\right)\right)= \\
& \quad\left(\left[\frac{1}{2}\left(k_{1}-k_{2}+1\right)\right]-\left[\frac{1}{4}\left(k_{1}-k_{2}+1\right)\right]\right) r\left(n_{1}\right)+\left(2\left[\frac{1}{4}\left(k_{1}-k_{2}+1\right)\right]-\left[\frac{1}{2}\left(k_{1}-k_{2}+1\right)\right]\right) r_{E}\left(n_{1}\right) .
\end{aligned}
$$

If $2 \gamma\left(k_{1}-k_{2}\right)$, then no Type II Lucas representations of $n$ can arise. In particular, if $k_{1}-k_{2} \equiv 3(\bmod 4)$, by simplifying the last formula, we obtain $r_{E}(n)=\frac{1}{4}\left(k_{1}-k_{72}+1\right) r\left(n_{1}\right)$. Similarly, if $k_{1}-k_{2} \equiv 1(\bmod 4)$, we obtain $r_{E}(n)=\frac{1}{4}\left(k_{1}-k_{2}+3\right) r\left(n_{1}\right)-r_{E}\left(n_{1}\right)$. If $2 \mid\left(k_{1}-k_{2}\right)$, we wish to count the number of Type II Lucas representations on $n$ that have evenly many terms. Each such representation originates from a Lucas representation of $n$ whose initial segment has $\frac{1}{2}\left(k_{1}-k_{2}\right)$ terms, and whose terminal segment's number of terms therefore differs in parity from $\frac{1}{2}\left(k_{1}-k_{2}\right)$. If $k_{1}-k_{2} \equiv 2(\bmod 4)$, then the number of Type II Lucas representations of $n$ is $\bar{r}_{E}\left(n_{1}\right)=r_{E}\left(n_{1}\right)-r_{0}\left(n_{2}\right)$, by Lemma 5 . In this case, the number of Type I Lucas representations of $n$ is $\frac{1}{4}\left(k_{1}-k_{2}+2\right)-r_{E}\left(n_{1}\right)$. Thus we obtain: $r_{E}(n)=\frac{1}{4}\left(k_{1}-k_{2}+2\right) r\left(n_{1}\right)-r_{0}\left(n_{2}\right)$. If $k_{1}-k_{2} \equiv 0(\bmod 4)$, then the number of Type II Lucas representations of $n$ is $\bar{r}_{0}\left(n_{1}\right)=r_{0}\left(n_{1}\right)-r_{E}\left(n_{2}\right)$, by Lemma 5 . In this case, the number of Type I Lucas representations of $n$ is $\frac{1}{4}\left(k_{1}-k_{2}\right) r\left(n_{1}\right)$. Therefore we obtain:

$$
r_{E}(n)=\frac{1}{4}\left(k_{1}-k_{2}\right) r\left(n_{1}\right)+r_{0}\left(n_{1}\right)-r_{E}\left(n_{2}\right)=\left(1+\frac{1}{4}\left(k_{1}-k_{2}\right)\right) r\left(n_{1}\right)-r_{E}\left(n_{1}\right)-r_{E}\left(n_{2}\right) .
$$

THEOREM 13. Let $n \neq L_{k}, n \neq L_{2 k+1}+1$. Then

$$
a(n)=\left\{\begin{array}{cc}
-a\left(n_{1}\right)-a\left(n_{2}\right) & \text { if } k_{1}-k_{2} \equiv 0(\bmod 4) \\
-a\left(n_{1}\right) & \text { if } k_{1}-k_{2} \equiv 1(\bmod 4) \\
a\left(n_{2}\right) & \text { if } k_{1}-k_{2} \equiv 2(\bmod 4) \\
0 & \text { if } k_{1}-k_{2} \equiv 3(\bmod 4)
\end{array} .\right.
$$

PROOF. This follows from (1.6) and from Theorems 11 and 12. 
LEMMA 6. If $n \geq 1$, then

$$
a\left(L_{n}-1\right)=\left\{\begin{aligned}
1 & \text { if } n \equiv 1(\bmod 4) \\
-1 & \text { if } n \equiv 2(\bmod 4) \\
0 & \text { if } n \equiv 0,3(\bmod 4)
\end{aligned}\right.
$$

PROOF. (Induction on $n$ ) The conclusion holds by inspection if $1 \leq n \leq 4$ If $n \geq 5$, then $L_{n}-1=L_{n-1}+\left(L_{n-2}+1\right)=L_{n-1}+L_{n-3}+\left(L_{n-4}-1\right)$. Now Theorem 13 implies $a\left(L_{n}-1\right)=$ $a\left(L_{n-4}-1\right)$, so the conclusion follows from the induction hypothesis.

LEMMA 7. If $n \geq 1$, then $a\left(2 L_{n}-1\right)=(-1)^{n}$.

PROOF. The conclusion holds by inspection if $1 \leq n \leq 4$. If $n \geq 5$, then $2 L_{n}-1=L_{n+1}+\left(L_{n-2}-1\right)=L_{n+1}+L_{n-3}+\left(L_{n-4}-1\right)$. Now Theorem 13 implies $a\left(2 L_{n}-1\right)=$ $-a\left(L_{n-2}-1\right)-a\left(L_{n-4}-1\right)$. The conclusion now follows from Lemma 6.

LEMMA 8. If $j \leq n \leq L_{k-3}-1$, then $a\left(2 L_{k}+n\right)=0$.

PROOF. $a\left(2 L_{k}+n\right)=a\left(L_{k+1}+L_{k-2}+n\right)$ by (1), so the conclusion follows from the hypothesis and Theorem 13.

LEMMA 9. If $0 \leq n \leq L_{k-2}-1$, then $a\left(5 F_{k}+n\right)=a(n)$.

PROOF. By (9), we have $a\left(5 F_{k}+n\right)=a\left(L_{k+1}+L_{k-1}+n\right)$. The conclusion now follows from the hypothesis and Theorem 13.

THEOREM 14. If $n$ belongs to $I_{k}$, where $k \geq 2$, and $m=L_{k+3}-1-n$, then (i) $r(m)=r(n)$ and (ii) $a(m)=(-1)^{k} a(n)$.

PROOF. It is easily seen that $m$ belongs to $I_{k}$ iff $n$ does. Now (7) implies there is a bijection between the partitions of $m, n$ respectively into distinct Lucas parts. Thus $r(m)=r(n)$. Furthermore, since the left side of (7) has $k+2$ terms, it follows that under this bijection, corresponding partitions of $m$ and $n$ will have numbers of parts that agree or disagree in parity accordingly as $k$ is even or odd. Therefore $a(m)=(-1)^{k} a(n)$.

COROLLARY 3. If $k \geq 1$, then $a\left(L_{4 k-2}-2\right)=-1 ; a\left(L_{4 k}-2\right)=2$.

PROOF. This follows from Theorems 10 and 14.

THEOREM 15. If $k \geq 3$, then $E\left(L_{k+2}-1\right)=E\left(L_{k+1}-1\right)+2 E\left(L_{k-2}-1\right)+2$.

PROOF. If $1 \leq i \leq 3 \leq k$, let $x_{k, i}=\Sigma\left\{|a(n)|: n \in I_{k, 2}\right\}$. Thus $x_{k, 1}+x_{k, 2}+x_{k, 3}=$ $E\left(L_{k+2}-1\right)-E\left(L_{k+1}-1\right)$. Now $x_{k, 3}=\Sigma\left\{|a(n)|: 5 F_{k} \leq n \leq L_{k+2}-1\right\}=E\left(L_{k+2}-1\right)-E\left(5 F_{k}-1\right)$. But Lemma 9 implies $a\left(5 F_{k}\right)=a(0)=1$, so $E\left(5 F_{k}\right)=1+E\left(5 F_{k}-1\right)$. Thus $x_{k, 3}=E\left(L_{k+2}-1\right)-$ $E\left(5 F_{k}\right)+1$. But (10) implies $L_{k+2}=5 F_{k}+L_{k-2}$, so $x_{k, 3}=E\left(5 F_{k}+L_{k-2}-1\right)-E\left(5 F_{k}\right)+1$. Now Lemma 9 implies $x_{k, 3}=E\left(L_{k-2}-1\right)+1$. Also, $x_{k, 2}=\Sigma\left\{|a(n)|: 2 L_{k} \leq n \leq 5 F_{k}-1\right\}=0$ by Lemma 8. Now $x_{k, 1}=\Sigma\left\{|a(n)|: L_{k+1} \leq n \leq 2 L_{k}-1\right\}$. Theorem 14 implies that $x_{k, 1}=\Sigma\left\{|a(n)|: 5 F_{k} \leq n \leq L_{k+2}-1\right\}=x_{k, 3}$. Thus we have: $E\left(L_{k+2}-1\right)-E\left(L_{k+1}-1\right)=$ $2\left(1+E\left(L_{k-2}-1\right)\right)$, from which the conclusion follows.

THEOREM 16. If $k \geq 2$ and if $L_{k+1} \leq n \leq L_{k+2}-1$, then

$$
E(n)= \begin{cases}E\left(2 L_{k}\right)-E\left(2 L_{k}-2-n\right)-1 & \text { if } L_{k+1} \leq n \leq 2 L_{k}-2 \\ E\left(2 L_{k}\right) & \text { if } 2 L_{k}-1 \leq n \leq 5 F_{k}-1 \\ E\left(2 L_{k}\right)+E\left(n-5 F_{k}\right)+1 & \text { if } 5 F_{k} \leq n \leq L_{k+2}-1\end{cases}
$$

PROOF. If $2 L_{k}-1 \leq n \leq 5 F_{k}-1$, then Lemma 8 implies $E(n)=E\left(2 L_{k}\right)$. If $5 F_{k} \leq n \leq L_{k+2}-1$, then $E(n)-E\left(5 F_{k}\right)=\sum_{j=1}^{n-5 F_{k}}\left|a\left(5 F_{k}+j\right)\right|=\sum_{j=1}^{n-5 F_{k}}|a(j)|=E\left(n-5 F_{k}\right)$ by Lemma 9. Also, Lemmas 8 and 9 imply $E\left(5 F_{k}\right)=1+E\left(2 L_{k}\right)$, so $E(n)=1+E\left(2 L_{k}\right)+E\left(n-5 F_{k}\right)$. Finally, if $L_{k+1} \leq n \leq 2 L_{k}-2$, let $m=2 L_{k}-n$, so that $2 \leq m \leq L_{k-2}$. We must show that $E\left(2 L_{k}-m\right)=E\left(2 L_{k}\right)-E(m-2)-1$. Now Lemmas 8 and 9 imply $a\left(2 L_{k}\right)=0$ and $\left|a\left(2 L_{k}-1\right)\right|=1$. Therefore $E\left(2 L_{k}\right)=E\left(2 L_{k}-1\right)=1+E\left(2 L_{k}-2\right)$. Thus it suffices to show that 
$E\left(2 L_{k}-m\right)=E\left(2 L_{k}-2\right)-E(m-2)$ when $2 \leq m \leq L_{k-2}$. This is trivially true when $m=2$. If $3 \leq m \leq L_{k-2}$, then $L_{k+1}+3 \leq 2 L_{k}-m \leq 2 L_{k}-3$, so that by (1), (9), Lemma 9 and Theorem 14, we have $\left|a\left(2 L_{k}-m\right)\right|=\left|a\left(L_{k+3}-1-2 L_{k}+m\right)\right|=\left|a\left(5 F_{k}+m-1\right)\right|=|a(m-1)|$. Therefore $E\left(2 L_{k}-2\right)-E\left(2 L_{k}-m\right)=\sum_{j=2}^{m-1}\left|a\left(2 L_{k}-j\right)\right|=\sum_{j=2}^{m-1}|a(j-1)|=\sum_{i=1}^{m-2}|a(i)|=E(m-2)$, so we are done.

THEOREM 17. $\lim _{n \rightarrow \infty} \frac{E(n)}{n}=0$.

PROOF. If $k \geq 2$, let $t_{k}=\max \left\{E(n) / n: n \in I_{k}\right\}$. It suffices to show that $\lim _{k \rightarrow \infty} t_{k}=0$. If $n \in I_{k}$, then by Theorem 16, we have: $E(n) \leq E\left(2 L_{k}\right)+E\left(n-5 F_{k}\right)+1$. Since $E(n)$ is non-decreasing and $n-5 F_{k} \leq L_{k-2}$, it follows that $E(n) \leq E\left(2 L_{k}\right)+E\left(L_{k-2}\right)+1$. By Theorem 16, we have $E\left(L_{k+1}\right)=E\left(2 L_{k}\right)-E\left(L_{k-2}-2\right)-1$, so we obtain $E(n) \leq E\left(L_{k+1}\right)+E\left(L_{k-2}\right)+E\left(L_{k-2}-2\right)+2$, hence $E(n) \leq E\left(L_{k+1}\right)+2 E\left(L_{k-2}\right)$. Since $n \leq L_{k+1}$, we get $\frac{E(n)}{n} \leq \frac{E\left(L_{k+1}\right)}{L_{k+1}}+2 \frac{E\left(L_{k-2}\right)}{L_{k+1}}$, so that $t_{k} \leq E\left(L_{k+1}\right) / L_{k+1}+2 E\left(L_{k-2}\right) / L_{k+1}$. Since $E(n)$ is non-decreasing and $L_{k}$ tends to infinity with $k$, it suffices to show that $\lim _{k \rightarrow \infty} E\left(L_{k}\right) / L_{k}=0$. In fact, since $E\left(L_{k}\right) \leq 1+E\left(L_{k}-1\right)$, it suffices to show that $\lim _{k \rightarrow \infty} E\left(L_{k}-1\right) / L_{k}=0$. If $k \geq 1$, let $c_{k}=E\left(L_{k}-1\right)$. Thus $c_{1}=0, c_{2}=c_{3}=2, c_{4}=4$. By Theorem 15, we have: $c_{k+2}=c_{k+1}+2 c_{k-2}+2$. Let the $\left\{c_{k}\right\}$ have the generating function: $F(z)=\sum_{k=1}^{\infty} c_{k} z^{k}$. Using the method of [2], p. 337-350, we obtain: $F(z)=\left(2 z^{2}-2 z^{3}+2 z^{4}\right) /\left(1-z^{2}\right)$ $\left(1-2 z+2 z^{2}-2 z^{3}\right)$. Therefore $c_{k}=b_{1} t_{1}^{k}+b_{2} t_{2}^{k}+b_{3} t_{3}^{k}+b_{4}+b_{5}(-1)^{k}$, where the $b_{i}$ are constants, and the $t_{i}$ are the roots of the equation: $x^{3}-2 x^{2}+2 x-2=0$. Using Cardan's formula, if $u=\frac{1}{3}(17+3 \sqrt{33})^{1 / 3}, v=\frac{1}{3}(17-3 \sqrt{33})^{1 / 3}$, then $t_{1}=\frac{2}{3}+u+v \doteq 1.544, t_{2}=\frac{2}{3}-\frac{1}{2}(u+v)+$ $\frac{i \sqrt{3}}{2}(u-v), t_{3}=\bar{t}_{2}$. Thus $\left|t_{2}\right|=\left|t_{3}\right| \doteq 1.138, b_{2}=b_{3}$, and $\left|b_{2} t_{2}^{k}+b_{3} t_{3}^{k}\right|=\left|2 b_{2} \operatorname{Re}\left(t_{2}^{k}\right)\right| \leq 2\left|b_{2}\right|\left|t_{2}^{k}\right|$. Now $0<\left|c_{k} / L_{k}\right| \leq\left(\left|b_{1}\right| 1.544^{k}+2\left|b_{2}\right| 1.138^{k}+\left|b_{4}\right|+\left|b_{5}\right|\right) / L_{k}$. But (6) implies that the right side of the last inequality tends to 0 as $k$ tends to infinity. Therefore $\lim _{k \rightarrow \infty} c_{k} / L_{k}=0$, we are done.

THEOREM 18. $a(n)$ assumes each of the values $0, \pm 1, \pm 2$ infinitely often.

PROOF. Theorem 7 implies $a(n)=0,-1$ infinitely often, while Theorem 10 implies $a(n)=2$ infinitely often. By Theorems 13 and $7, a\left(L_{4 k+5}+L_{4 k}\right)=-a\left(L_{4 k}\right)=1$. Therefore $a(n)=1$ infinitely often. Finally, with $k \geq 2$, let $n=L_{4 k}+5=L_{4 k}+L_{3}+L_{1}$. Now Theorem 13 implies $a(n)=-a(5)=-2$. Therefore $a(n)=-2$ infinitely often.

THEOREM 19. $|a(n)| \leq 2$ for all $n$.

PROOF. If $|a(n)| \geq 3$ for some $n$, let $n$ be the least such integer. By Theorems 7 and $10, n \neq L_{k}$, $L_{2 k+1}+1$. Let $n$ have the minimal Lucas representation:

$$
n=n_{0}=L_{k_{1}}+L_{k_{2}}+\text { etc. }+L_{k_{r}} \text { where } r \geq 2 ; \text { let } n_{i}=n_{2-1}-L_{k_{1}}
$$

for $1 \leq i \leq r$, with $n_{r}=0$. By hypothesis and Theorem 13, we must have: $a(n)=-\left(a\left(n_{1}\right)+a\left(n_{2}\right)\right)$, with $k_{1}-k_{2} \equiv 0(\bmod 4)$. By Theorem 13 implies

$$
a\left(n_{1}\right)+a\left(n_{2}\right)=\left\{\begin{array}{ll}
-a\left(n_{3}\right) & \text { if } k_{2}-k_{3} \equiv 0(\bmod 4) \\
0 & \text { if } k_{2}-k_{3} \equiv 1(\bmod 4) \\
a\left(n_{2}\right)+a\left(n_{3}\right) & \text { if } k_{2}-k_{3} \equiv 2(\bmod 4) \\
a\left(n_{2}\right) & \text { if } k_{2}-k_{3} \equiv 3(\bmod 4)
\end{array} .\right.
$$

Therefore $a(n)=-\left(a\left(n_{2}\right)+a\left(n_{3}\right)\right)$, with $k_{2}-k_{3} \equiv 2(\bmod 4)$. If we apply Theorem 13 repeatedly, we eventually get $a(n)=-\left(a\left(n_{r-1}\right)+a\left(n_{r}\right)\right)=-\left(a\left(L_{k_{r}}\right)+a(0)\right)$. Now Theorem 7 implies $|a(n)| \leq 1$, contrary to hypothesis.

ACKNOWLEDGMENT. Theorems 14 through 17 are Lucas analogues of results about Fibonacci partitions announced by Weinstein in [4]. For each integer $n$, such that $0 \leq n \leq 100$, Table 1 lists $r(n), r_{E}(n), a(n), E(n)$, and $V(n)$. 
Table 1

\begin{tabular}{|c|c|c|c|c|c|c|c|c|c|c|c|}
\hline$\underline{n}$ & $r(n)$ & $r_{E}(n)$ & $a(n)$ & $E(n)$ & $V(n)$ & $n$ & $r(n)$ & $r_{E}(n)$ & $a(n)$ & $E(n)$ & $V(n)$ \\
\hline 0 & 1 & 1 & 1 & 0 & 1 & 51 & 6 & 3 & 0 & 30 & 6308 \\
\hline 1 & 1 & 0 & -1 & 1 & 1 & 52 & 6 & 2 & -2 & 32 & 6877 \\
\hline 2 & 1 & 0 & -1 & 2 & 2 & 53 & 6 & 3 & 0 & 32 & 7491 \\
\hline 3 & 2 & 1 & 0 & 2 & 3 & 54 & 8 & 4 & 0 & 32 & 8155 \\
\hline 4 & 2 & 1 & 0 & 2 & 5 & 55 & 5 & 3 & 1 & 33 & 8862 \\
\hline 5 & 2 & 2 & 2 & 4 & 6 & 56 & 5 & 3 & 1 & 34 & 9622 \\
\hline 6 & 2 & 1 & 0 & 4 & 9 & 57 & 7 & 3 & -1 & 35 & 10438 \\
\hline 7 & 3 & 1 & -1 & 5 & 12 & 58 & 6 & 3 & 0 & 35 & 11316 \\
\hline 8 & 2 & 1 & 0 & 5 & 16 & 59 & 6 & 3 & 0 & 35 & 12247 \\
\hline 9 & 2 & 1 & 0 & 5 & 20 & 60 & 6 & 3 & 0 & 35 & 13249 \\
\hline 10 & 3 & 2 & 1 & 6 & 26 & 61 & 6 & 3 & 0 & 35 & 14319 \\
\hline 11 & 3 & 1 & -1 & 7 & 33 & 62 & 6 & 3 & 0 & 35 & 15464 \\
\hline 12 & 3 & 1 & -1 & 8 & 41 & 63 & 6 & 3 & 0 & 35 & 16678 \\
\hline 13 & 3 & 2 & 1 & 9 & 50 & 64 & 6 & 3 & 0 & 35 & 17981 \\
\hline 14 & 4 & 2 & 0 & 9 & 62 & 65 & 7 & 4 & 1 & 36 & 19369 \\
\hline 15 & 3 & 2 & 1 & 10 & 75 & 66 & 5 & 2 & -1 & 37 & 20845 \\
\hline 16 & 3 & 1 & -1 & 11 & 90 & 67 & 5 & 2 & -1 & 38 & 22413 \\
\hline 17 & 3 & 1 & -1 & 12 & 107 & 68 & 8 & 4 & 0 & 38 & 24089 \\
\hline 18 & 4 & 2 & 0 & 12 & 129 & 69 & 6 & 3 & 0 & 38 & 25868 \\
\hline 19 & 3 & 2 & 1 & 13 & 151 & 70 & 6 & 4 & 2 & 40 & 27754 \\
\hline 20 & 3 & 2 & 1 & 14 & 178 & 71 & 6 & 3 & 0 & 40 & 29759 \\
\hline 21 & 5 & 2 & -1 & 15 & 208 & 72 & 7 & 3 & -1 & 41 & 31893 \\
\hline 22 & 4 & 2 & 0 & 15 & 244 & 73 & 4 & 2 & 0 & 41 & 34149 \\
\hline 23 & 4 & 2 & 0 & 15 & 281 & 74 & 4 & 2 & 0 & 41 & 36541 \\
\hline 24 & 4 & 2 & 0 & 15 & 326 & 75 & 5 & 3 & 1 & 42 & 39078 \\
\hline 25 & 5 & 3 & 1 & 16 & 375 & 76 & 7 & 3 & -1 & 43 & 41771 \\
\hline 26 & 3 & 1 & -1 & 17 & 431 & 77 & 5 & 2 & -1 & 44 & 44609 \\
\hline 27 & 3 & 1 & -1 & 18 & 491 & 78 & 5 & 3 & 1 & 45 & 47619 \\
\hline 28 & 4 & 2 & 0 & 18 & 561 & 79 & 8 & 4 & 0 & 45 & 50802 \\
\hline 29 & 4 & 2 & 0 & 18 & 638 & 80 & 7 & 4 & 1 & 46 & 54170 \\
\hline 30 & 4 & 3 & 2 & 20 & 723 & 81 & 7 & 3 & -1 & 47 & 57715 \\
\hline 31 & 4 & 2 & 0 & 20 & 816 & 82 & 7 & 3 & -1 & 48 & 61471 \\
\hline 32 & 6 & 3 & 0 & 20 & 922 & 83 & 9 & 5 & 1 & 49 & 65434 \\
\hline 33 & 5 & 2 & -1 & 21 & 1037 & 84 & 6 & 3 & 0 & 49 & 69613 \\
\hline 34 & 5 & 2 & -1 & 22 & 1163 & 85 & 6 & 3 & 0 & 49 & 74013 \\
\hline 35 & 5 & 3 & 1 & 23 & 1302 & 86 & 7 & 3 & -1 & 50 & 78664 \\
\hline 36 & 6 & 3 & 0 & 23 & 1458 & 87 & 8 & 4 & 0 & 50 & 83561 \\
\hline 37 & 4 & 2 & 0 & 23 & 1624 & 88 & 8 & 5 & 2 & 52 & 88715 \\
\hline 38 & 4 & 2 & 0 & 23 & 1808 & 89 & 8 & 4 & .0 & 52 & 94140 \\
\hline 39 & 6 & 3 & 0 & 23 & 2009 & 90 & 6 & 3 & 0 & 52 & 99862 \\
\hline 40 & 5 & 3 & 1 & 24 & 2231 & 91 & 7 & 3 & -1 & 53 & 105871 \\
\hline 41 & 5 & 2 & -1 & 25 & 2467 & 92 & 7 & 3 & -1 & 54 & 112190 \\
\hline 42 & 5 & 2 & -1 & 26 & 2729 & 93 & 7 & 4 & 1 & 55 & 118835 \\
\hline 43 & 6 & 3 & 0 & 26 & 3012 & 94 & 8 & 4 & 0 & 55 & 125830 \\
\hline 44 & 4 & 2 & 0 & 26 & 3321 & 95 & 6 & 3 & 0 & 55 & 133160 \\
\hline 45 & 4 & 3 & 2 & 28 & 3651 & 96 & 6 & 3 & 0 & 55 & 140867 \\
\hline 46 & 4 & 2 & 0 & 28 & 4014 & 97 & 10 & 5 & 0 & 55 & 148958 \\
\hline 47 & 5 & 2 & -1 & 29 & 4406 & 98 & 8 & 4 & 0 & 55 & 157456 \\
\hline 48 & 4 & 2 & 0 & 29 & 4828 & 99 & 8 & 4 & 0 & 55 & 166353 \\
\hline 49 & 4 & 2 & 0 & 29 & 5282 & 100 & 8 & 4 & 0 & 55 & 175400 \\
\hline 50 & 7 & 4 & 1 & 30 & 5777 & & & & & & \\
\hline
\end{tabular}

\section{REFERENCES}

[1] APOSTOL, T.M., Introduction to Analytic Number Theory, Springer Verlag, 1976.

[2] GRAHAM, R.L., KNUTH, D.E. and PATASHNIK, O., Concrete Mathematics, Addison-Wesley, 2nd Ed., 1994.

[3] HARDY, G.H. and WRIGHT, E.M., An Introduction to the Theory of Numbers, Oxford University Press, 4th Ed., 1960.

[4] WEINSTEIN, F.V., private communication. 


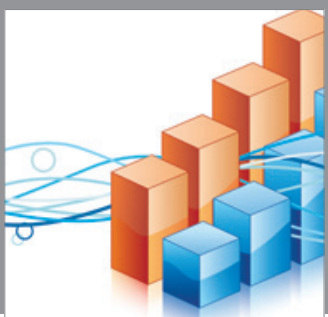

Advances in

Operations Research

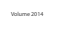

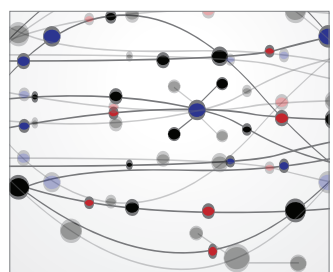

\section{The Scientific} World Journal
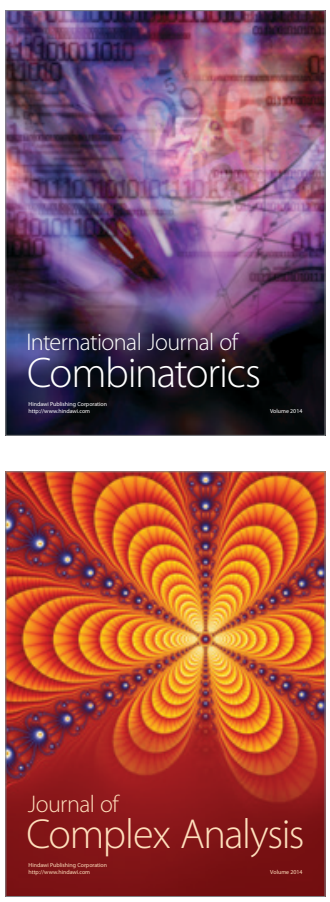

International Journal of

Mathematics and

Mathematical

Sciences
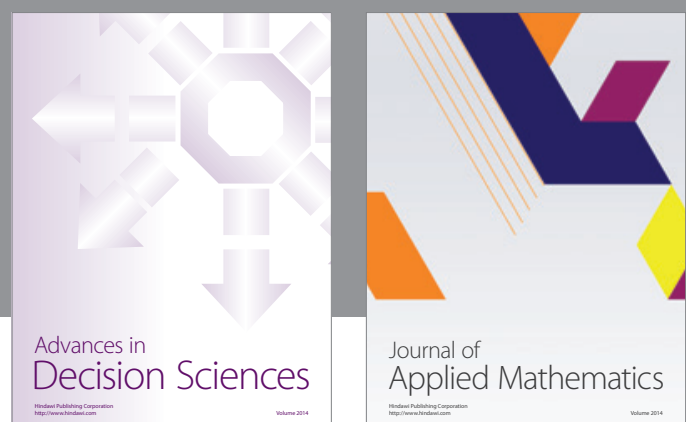

Journal of

Applied Mathematics
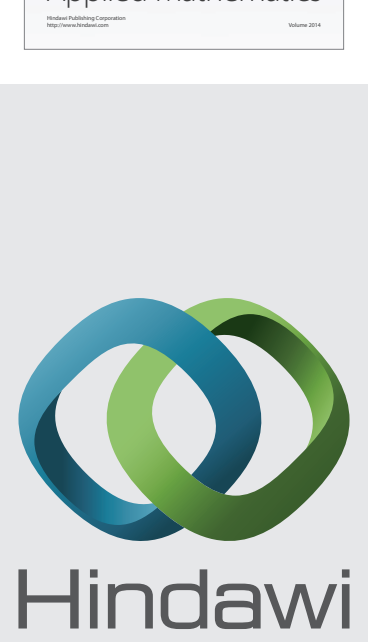

Submit your manuscripts at http://www.hindawi.com
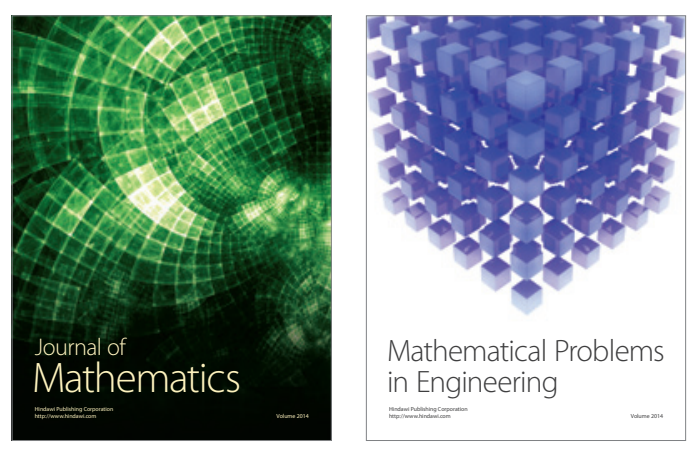

Mathematical Problems in Engineering
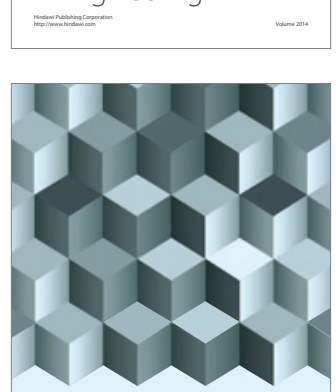

Journal of

Function Spaces
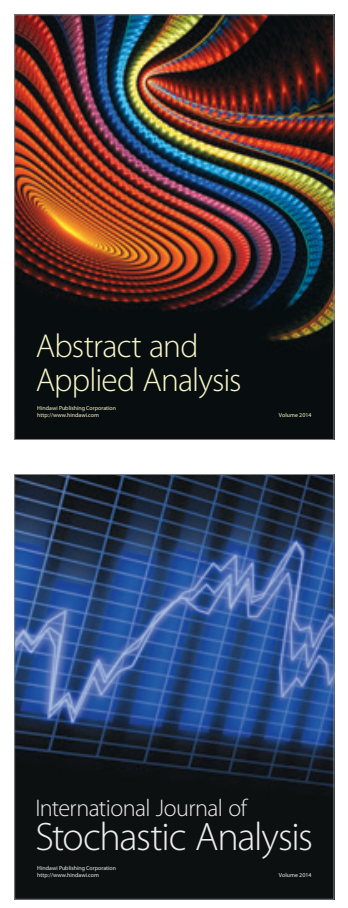

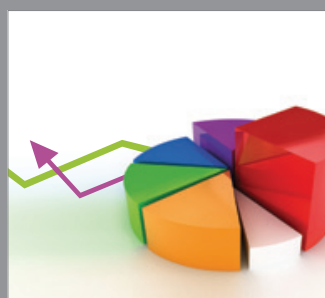

ournal of

Probability and Statistics

Promensencen
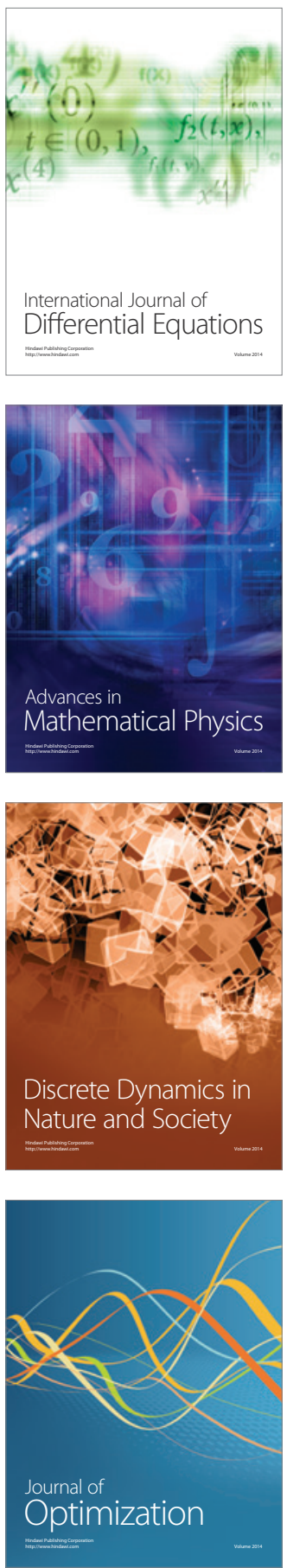